\title{
Was beeinflusst die Einstellungen von Schülerinnen und Schülern zum Lernen mit digitalen Medien? Eine Analyse der Befragungen von PISA 2012 in der Schweiz
}

\section{Dominik Petko, Andrea Cantieni und Doreen Prasse}

Schülerinnen und Schüler sollen digitale Technologien in der Schule nicht nur als Lernwerkzeug kennenlernen, sondern idealerweise auch positive Einstellungen dazu entwickeln. In welchem Ausmass Schule hierzu tatsächlich einen Beitrag leistet, wird anhand der Schweizer Teilstichprobe der Schülerinnen-und Schülerbefragungen von PISA-Studie 2012 geprüft. Die Mehrebenenanalysen zeigen, dass insbesondere die häusliche Nutzung digitaler Medien für schulische Zwecke einen Zusammenhang mit den Einstellungen der Schülerinnen und Schüler hat, während die schulische Nutzung nur einen kleinen Effekt besitzt. Dieser Effekt kann unter Umständen mit der vergleichsweise seltenen schulischen Mediennutzung in der Schweiz erklärt werden. Im Sinne einer Chancengleichheit wird dafür plädiert, dass Schulen hier aktiver werden müssen.

\section{Einleitung}

Die Rolle digitaler Technologien für Lehr- und Lernzwecke ist seit den ersten PISA Studien ein zentraler Fokus der Schülerinnen- und Schülerbefragungen. Seit dem Jahr 2000 liefern die PISA Studien im dreijährigen Turnus international vergleichbare Daten zur Computerausstattung in Schulen, zur Häufigkeit der Nutzung verschiedener digitaler Technologien für schulische und ausserschulische Zwecke sowie zu selbsteingeschätzten Kompetenzen und Überzeugungen der Schülerinnen im Bereich dieser Technologien (OECD, 2015; Shewbridge, Ikeda \& Schleicher, 2005). Für die Schweiz und Deutschland dokumentierte PISA dabei immer wieder, dass die Einführung der neuen Geräte in den meisten Schulen im Vergleich zu anderen Ländern eher schleppend verlief, insbesondere in der Westschweiz (Huber \& Ramseier, 2002; Ramseier \& Holzer, 2005; Salvisberg, 2014). Die Schweiz gehörte - ebenso wie Deutschland - immer wieder zu den Ländern mit der geringsten durchschnittlichen Nutzung digitaler Medien im Unterricht, trotz überdurchschnittlicher Ausstattung. Ausserdem ergab sich in der Schweiz regelmässig eine der grössten Scheren zwischen inten- 
siver häuslicher und seltener schulischer Computernutzung. Dennoch schätzten sich Schweizer Schülerinnen und Schüler als sehr kompetent im Umgang mit digitalen Medien ein und zeigten auch, wie in praktisch allen Ländern, eine mehrheitlich positive Einstellung zum Umgang mit digitalen Geräten. Neben solchen deskriptiven Befunden interessierte in PISA Studien aber vor allem auch die Frage, in welchem Zusammenhang Computernutzung und fachliche Leistungen - gemessen an den Testergebnissen in der Muttersprache, Mathematik und Naturwissenschaften - stehen.

\section{Einstellungen zur Qualität schulbezogener Computernutzung als Prädiktor für fachspezifische \\ Testleistungen}

Mit dem Einsatz digitaler Medien war insbesondere in der Pionierzeit, in der in Schulen viel Geld in den Aufbau der Infrastruktur investiert wurde, die allgemeine Erwartung verbunden, dass dies zu einer Verbesserung des Unterrichts und einer Steigerung des Leistungsniveaus in Schulen führen würde. Die bisherigen Studien können dies jedoch noch nicht bestätigen. Zwar ist eine häufige häusliche Computernutzung in den meisten Ländern durchaus ein positiver Prädiktor für Testleistungen, eine häufige schulische Computernutzung scheint jedoch oft zumindest nach den Ergebnissen von Large-Scale Studien - entweder kein oder sogar ein negativer Faktor zu sein, auch unter Berücksichtigung verschiedener Kontrollvariablen (Gerick \& Eickelmann, 2015; OECD, 2015; Shewbridge et al., 2005). Ähnliche Ergebnisse finden sich auch bei anderen grossen Schulleistungsvergleichen wie PIRLS und TIMSS (House, 2007; Lorenz \& Gerick, 2014; Papanastasiou \& Paparistodemou, 2007; Skryabin, Zhang, Liu \& Zhang, 2015). Viele Studien mahnen auf Basis dieser ernüchternden Befunde zu Vorsicht vor Technikeuphorie und raten teilweise sogar von einer allzu häufigen Nutzung digitaler Technologien im Unterricht ab (OECD, 2015; Woessmann \& Fuchs, 2007). Solche Befunde aus Large-Scale-Studien sind umso erstaunlicher als dass sich in experimentellen Untersuchungen viele positive Effekte digitaler Technologien nachweisen lassen, wie Meta-Analysen immer wieder zeigen (Cheung \& Slavin, 2012, 2013; Reimann \& Aditomo, 2013; Tamim, Bernard, Borokhovski, Abrami \& Schmid, 2011). Es lassen sich jedoch auch verschiedene Gründe anführen, die Vorsicht vor einer vorschnellen Interpretation dieser Befunde nahelegen. Erstens ist der Untersuchungsgegenstand «digitale Medien» äusserst uneinheitlich, da sich hinter diesem Sammelbegriff unterschiedlichste Technologien und Anwendungsmöglichkeiten verbergen. Zweitens erfolgten die Leistungsmessungen bei PISA bisher überwiegend mit traditionellem Paperand-Pencil Verfahren, die mögliche digitale Kompetenzen nicht einschlossen. In den neuesten PISA Kohorten, die in einigen Ländern bereits 2012 auch mithilfe von computerunterstützten Testverfahren getestet wurden, scheint sich der negative Zusammenhang zwischen schulischer Computernutzung und fachlicher Testleistung jedoch zu bestätigen (OECD, 2015). Drittens handelt 
es sich bei PISA, TIMSS und PIRLS durchgängig um Querschnittsuntersuchungen, die lediglich korrelative Auswertungen zulassen, die nicht unbedingt kausal interpretiert werden können. Der vermutlich wichtigste Grund für die eingeschränkte Aussagekraft dieser Studien zum Zusammenhang von fachspezifischer Testleistung und ICT-Nutzung liegt jedoch im Umstand, dass im Rahmen von Large-Scale-Studien bisher vor allem die Quantität und nicht die Qualität der schulischen Computernutzung untersucht wurde. Typischerweise wurde beispielsweise abgefragt, wie häufig die eine oder andere Hardware oder Software im Unterricht eingesetzt wurde, nicht aber, mit welchem Lernziel, mit welchem didaktischen Kontext und mit welchem Erfolg. Einen Neuansatz bietet demgegenüber die Studie von Petko, Cantieni und Prasse (2016), die mit den Schülerinnen- und Schülerdaten von PISA 2012 zeigen konnte, dass nicht die Quantität, sondern die Einstellungen zur wahrgenommenen Qualität der ICT-Nutzung für Lernzwecke in praktisch allen Bildungssystemen einen deutlichen und positiven Prädiktor für Testleistungen in den verschiedenen Fächern darstellt. Schon kamen einzelne Studien zu dem Schluss, dass die Einstellungen von Schülerinnen und Schülern gegenüber digitalen Technologien einen positiven Zusammenhang mit ihren fachlichen Testleistungen besitzen (Lee \& Wu, 2012). Im Vergleich zu diesen Studien liegt der Vorteil der Daten von PISA 2012 darin (OECD, 2014), dass hier die Items erstmals so formuliert wurden, dass die Einstellungen einen direkten Zusammenhang mit spezifisch schulischen Nützlichkeitserfahrungen haben (Beispielitem für die Skala ICTATTPOS in PISA 2012: "The computer is a very useful tool for my schoolwork»). So kann geschlussfolgert werden, dass bei Schülerinnen und Schülern, die solche Items positiv beurteilen, wahrscheinlich auch positive Lernerfahrungen mit digitalen Medien vorhanden sind. Damit können die Einstellungen der Schülerinnen und Schüler als mittelbares Mass der Qualität der erlebten Lernerfahrungen mit digitalen Medien interpretiert werden. Gleichzeitig sind gewisse Zweifel bei der Richtung und der Konfundierung des beobachteten Zusammenhangs angebracht. So ist durchaus denkbar, dass sich bei leistungsstärkeren Schülerinnen und Schülern generell positivere Einstellungen zum schulischen Lernen finden und sich dies auch in den Einstellungen zum Lernen mit digitalen Technologien spiegelt. $\mathrm{Ob}$ und zu welchem Grad sich die diesbezüglichen Einstellungen auf spezifisch schulische und ausserschulische Medienerfahrungen zurückführen lassen, ist noch ungeklärt. PISA 2015 (OECD, 2017) ermöglicht zur Klärung dieser Fragen einerseits Fortschritte, da neu durchgängig mit computerbasierten Testaufgaben gearbeitet wird und damit auch Kompetenzen zur Nutzung digitaler Medien für Lernzwecke mitgetestet werden, andererseits aber auch Rückschritte, da in den Begleitbefragungen zu computerbezogenen Einstellungen nicht mehr auf lernbezogene, sondern wieder nur auf allgemeine Einstellungen und Selbstwirksamkeitsüberzeugungen Bezug genommen wird. Bisher liegen keine Befunde zu PISA 2015 vor, die analysieren, ob die selbsteingeschätzten digitalen Kompetenzen und Überzeugungen einen stärkeren 
Einfluss auf die Testleistungen haben. In den Piloterhebungen der digitalen Testungen bei PISA 2012 zeigten sich hier jedoch bereits vereinzelt stärkere Einflüsse (Naumann \& Sälzer, 2017; OECD, 2015). Bei PISA 2015 zeigen erste Ergebnisse ausserdem, dass sich die computerbasierten Mathematiktests nur in Ländern mit extrem hoher Computernutzung im Unterricht in einem Trend zu besseren Testleistungen niederschlagen (OECD, 2016).

\section{Einstellungen zur Qualität schulbezogener Computer- nutzung als Prädiktor für künftige Nutzung digitaler Technologien und deren Prämissen}

Die Einstellungen von Schülerinnen und Schülern zum Lernen mit digitalen Medien sind allerdings nicht nur als Hinweis auf die Qualität des schulischen und ausserschulischen Medieneinsatzes und als Prädiktor für Testleistungen bedeutsam, sondern sie stellen auch für sich genommen ein bedeutsames Lernziel dar (Delors, 1998; Rychen \& Salganik, 2003). Wenn Schülerinnen und Schüler positive Einstellungen zum Lernen mit digitalen Medien entwickeln, dann sind sie wahrscheinlich auch motivierter, künftig digitale Technologien verstärkt als Lerninstrument einzusetzen. Eine solche Interpretation legen Theorien wie die Theory of Reasoned Action oder die Theory of Planned Behavior nahe (Fishbein \& Ajzen, 1977, 2010), oder noch spezifischer, daraus abgeleitete Technology Acceptance Modelle (Davis, 1989; Venkatesh, Thong \& Xu, 2012), in denen die Einstellungen eine zentrale Rolle zur Vorhersage künftigen Verhaltens einnehmen. Entscheidend sind hierbei vor allem die wahrgenommene Nützlichkeit («perceived usefulness») und die einfache Nutzbarkeit («perceived ease-of-use») einer bestimmten technologischen Innovation. Gerade letztere Einschätzung besitzt wiederum einen engen Zusammenhang mit der technologiebezogenen Selbstwirksamkeit. Konkrete Studien zur Bedeutung technologiebezogener Einstellungen und Überzeugungen von Schülerinnen und Schülern zum Lernen mit digitalen Medien sind jedoch noch ebenso rar, wie Studien zur Genese dieser Einstellungen. Lange schien einfach angenommen zu werden, dass die Generation der «Digital Natives» selbstredend positive Einstellungen zu digitalen Technologien aufweise und ganz selbstverständlich damit lerne (Oblinger \& Oblinger, 2005; Tapscott, 1998). Solche Annahmen können mittlerweile als widerlegt gelten und es wird verstärkt eine differenzielle Sicht auf diese Einstellungen angemahnt (Jones, Ramanau, Cross \& Healing, 2010; Schulmeister, 2009). Während z.B. schon einige Forschung dazu besteht, die zeigen konnte, dass deutliche Unterschiede in den Einstellungen bezüglich des Geschlechts und des sozioökonomischen Status bestehen, sind darüber hinausgehende Analysen noch relativ unklar (Meelissen, 2008; Young, 2000). Neuerdings werden computerbezogene Einstellungen deshalb zunehmend auch in umfassenderer Weise als abhängige Variable untersucht (Hatzigianni, Gregoriadis \& Fleer, 2016; Howard, Ma \& Yang, 2016; Prasse \& Egger, 2016, Juni). Die bisherigen Resultate deuten dabei darauf hin, dass schulische Computer- 
nutzung durchaus einen positiven Einfluss auf die Einstellungen von Schülerinnen und Schülern zu digitalen Medien haben kann, jedoch wurde dies bisher noch kaum im Zusammenspiel mit der häuslichen Mediennutzung untersucht. So lassen beispielsweise die Ergebnisse von Prasse, Egger und Döbeli Honegger (2017) im Rahmen einer Studie zur Tabletnutzung von Primarschülerinnen und -schülern einen Zusammenhang zwischen häuslicher lernbezogener Nutzung digitaler Medien und Einstellungen vermuten. Hier setzt die vorliegende Studie an. Ausgehend von der Annahme, dass positive medienbezogene Einstellungen von Schülerinnen und Schülern ein Ziel pädagogischer Aktivitäten darstellen können, stellt sich die Anschlussfrage, von welchen Faktoren die Einstellungen von Schülerinnen und Schüler zur Mediennutzung ihrerseits beeinflusst werden. Die allgemeine Forschungsliteratur zur Genese von Einstellungen (d.h. von "attitudes", die eine mehr oder weniger positive Bewertung zu einem bestimmten Sachverhalt beinhalten) und Überzeugungen (d.h. «beliefs», bei denen es eher um Aussagen geht, die subjektiv für wahr oder falsch gehalten werden) zeigt eindrucksvoll die Komplexität möglicher Einflussfaktoren, die von praktischen Erfahrungen, überzeugenden Argumenten relevanter Bezugspersonen bis hin zu latenten Werthaltungen innerhalb sozialer Kontexte reichen (Bohner \& Dickel, 2011). Die oben genannten «Technology Acceptance Models» gehen ebenfalls davon aus, dass Einstellungen zur Nützlichkeit und zur Nutzbarkeit von Technologien von einer Vielzahl weiterer Faktoren beeinflusst werden, darunter das Alter, das Geschlecht und die konkreten Medienerfahrungen und Mediengewohnheiten, ausserdem die Einstellungen relevanter Bezugspersonen und Bezugsgruppen, Zugang zu Geräten und Kosten und nicht zuletzt auch emotionale Facetten wie Spass und Befriedigung von Bedürfnissen. Welchen Einfluss dabei schulische Medienerfahrungen haben, ist bisher noch unklar. Während manche Studien von deutlichen Zusammenhängen berichten (Levin \& Wadmany, 2005), berichten andere eher davon, dass sich die diesbezüglichen Einstellungen von Schülerinnen und Schülern unabhängig von den unterrichtlichen Medienerfahrungen und den diesbezüglichen Intentionen von Lehrpersonen entwickeln (Li, 2007). Vor diesem Hintergrund muss gefragt werden, welchen Einfluss schulische und häusliche Medienpraktiken neben Kontrollvariablen wie dem Geschlecht und dem sozioökonomischen Status, die in internationalen Schulleistungsvergleichen zumindest in Bezug auf die schulischen Leistungen wesentliche Prädiktoren darstellen, auf die medienbezogenen Einstellungen von Schülerinnen und Schülern besitzen.

\section{Fragestellungen und Hypothesen}

Nachdem in den Analysen von Petko et al. (2016) gezeigt wurde, dass positive Einstellungen zur Nutzung digitaler Medien als Lerninstrument einen deutlichen und positiven Einfluss auf Testleistungen in PISA 2012 besitzen 
und davon ausgegangen werden kann, dass positive Einstellungen zum Lernen mit digitalen Technologien eine wünschenswerte Voraussetzung für künftiges Verhalten darstellen, soll hier der weiterführenden Frage nachgegangen werden, welche Einflussfaktoren entscheidend für diese positiven Einstellungen sind. Dabei soll die Frage im Mittelpunkt stehen, welchen Einfluss in dieser Hinsicht die schulische Nutzung digitaler Medien, die häusliche Nutzung für schulische Zwecke und die häusliche Nutzung für Freizeitzwecke haben. Als Kontrollvariablen müssen die schulische und häusliche Medienverfügbarkeit berücksichtigt werden, da eine ausreichende Zahl zugänglicher Geräte die Grundvoraussetzung für ihren Einsatz darstellt. Als allgemeine Kontrollvariablen dienen zudem das Geschlecht und der sozioökonomische Status. Im Einzelnen werden folgende Hypothesen geprüft:

H1 Die schulbezogene Nutzung digitaler Medien a) in der Schule und b) zuhause ist ein signifikanter und positiver Prädiktor für die diesbezüglichen Einstellungen der Schülerinnen und Schüler.

H2 Die freizeitbezogene Nutzung digitaler Medien zuhause ist ein signifikanter Prädiktor für die Einstellungen von Schülerinnen und Schülern bezüglich des Lernens mit digitalen Medien.

H3 Schulen unterscheiden sich in der Stärke dieser Zusammenhänge, so dass ein Teil der individuellen Varianz auf die Zugehörigkeit der Schülerinnen und Schüler zu einer bestimmten Schule zurückzuführen ist.

\section{Methoden}

\section{St ichprobe}

Die Hypothesen werden anhand des Schweizer Schülerdatensatzes von PISA 2012 geprüft. PISA ist vermutlich der mit Abstand bekannteste internationale Schulleistungsvergleich, bei dem seit dem Jahr 2000 in dreijährigem Turnus im Auftrag der OECD eine repräsentative Stichprobe von 15-jährigen Schülerinnen und Schülern in den teilnehmenden Kantonen befragt und in schulischen Kernfächern vergleichend getestet wird. Bei PISA 2012 wurden gut 510`000 Schülerinnen und Schüler aus 65 Ländern bzw. Bildungssystemen untersucht. Standardmässig werden angewandte Kompetenzen in der primären Unterrichtssprache, Mathematik und Naturwissenschaften getestet. Darüber hinaus werden auch ergänzende Testoptionen angeboten, an denen allerdings nicht alle Länder teilnehmen. Neben den Tests werden auch Begleitbefragungen durchgeführt, u.a. spezielle Vertiefungsoptionen zum Bereich der Informationsund Kommunikationstechnologien (ICT). Bei PISA 2012 beteiligten sich 40 der 65 Bildungssysteme an dieser ICT-Option der Schülerinnen- und Schülerbefragung. Die Schweiz ist seit dem Jahr 2000 sowohl an den PISA Studien und auch an der Option des «Information and Communication Technology Familiarity Questionnaire» beteiligt. Die Daten von PISA 2012 sind öffentlich 
verfügbar (http://pisa2012.acer.edu.au) und im Detail dokumentiert (OECD, 2014). Die Schweizer Teilstichprobe des internationalen Datensatzes von PISA 2012 umfasst $\mathrm{N}=11^{\prime} 229$ Schülerinnen und Schüler im Alter von 15 Jahren, von denen $50.32 \%$ männlich und $49.68 \%$ weiblich sind. Die Schülerinnen und Schüler stammen aus gesamthaft 411 Schulen, aus allen Landesteilen und aus allen Schulformen. Daneben existiert noch eine nationale Erweiterungsstichprobe der 9. Klassen, die auch sprachregionale Vergleiche zulässt, die für diese Studie jedoch nicht berücksichtigt wird.

\section{Variablen}

Die folgende Tabelle 1 zeigt die Variablen aus dem allgemeinen Schülerfragebogen und dem «Information and Communication Technology Familiarity Questionnaire» von PISA 2012, die für die Prüfung der Hypothesen berücksichtigt werden.

Tabelle 1: Beispielitems, Skalenwerte und Skalenreliabilitäten für die Schweizer Stichprobe, gewichtet mit Final Student Weight (W_FSTUWT)

\begin{tabular}{|c|c|c|c|c|}
\hline & Beispielitem & Antwortformat & $\mathrm{M}(\mathrm{SD})$ & $\mathrm{C} \alpha$ \\
\hline ST04Q01 & Geschlecht & weiblich / männlich & - & - \\
\hline ESCS & $\begin{array}{l}\text { PISA Index des ökonomischen, } \\
\text { sozialen und kulturellen Status }\end{array}$ & $\begin{array}{l}\text { Kompositskala verschie- } \\
\text { dener Indizes }\end{array}$ & $.17(.89)$ & .65 \\
\hline ICTATTPOS & $\begin{array}{l}\text { Der Computer ist sehr nützlich } \\
\text { für schulische Aufgaben. }\end{array}$ & $\begin{array}{l}\text { "stimmt völlig”, ,stimmt } \\
\text { eher”, ,stimmt eher nicht”, } \\
\text { „stimmt überhaupt nicht" }\end{array}$ & $-.34(1.03)$ & .75 \\
\hline ICTHOME & $\begin{array}{l}\text { Sind die folgenden Dinge für } \\
\text { dich zuhause verfügbar? Ein } \\
\text { Computer }\end{array}$ & $\begin{array}{l}\text { Ja, und ich benütze sie } \\
\text { auch / Ja, aber ich benütze } \\
\text { sie nicht / Nein }\end{array}$ & $.30(.81)$ & .47 \\
\hline ICTSCH & $\begin{array}{l}\text { Sind die folgenden Dinge für } \\
\text { dich in der Schule verfügbar? Ein } \\
\text { Computer }\end{array}$ & $\begin{array}{l}\text { Ja, und ich benütze sie } \\
\text { auch / Ja, aber ich benütze } \\
\text { sie nicht / Nein }\end{array}$ & $.18(.92)$ & .66 \\
\hline ENTUSE & $\begin{array}{l}\text { Wie oft benutzt du außerhalb } \\
\text { der Schule den Computer für } \\
\text { folgende Aktivitäten? Spiele im } \\
\text { Einzelspieler-Modus }\end{array}$ & $\begin{array}{l}\text { Nie oder fast nie / ein- } \\
\text { oder zweimal im Monat / } \\
\text { ein- oder zweimal in der } \\
\text { Woche / fast jeden Tag / } \\
\text { jeden Tag }\end{array}$ & $-.06(.84)$ & .77 \\
\hline $\mathrm{HOMSCH}$ & $\begin{array}{l}\text { Wie oft benutzt du außerhalb } \\
\text { der Schule den Computer } \\
\text { für folgende Aktivitäten? Für } \\
\text { schulische Aufgaben im Internet } \\
\text { surfen (z. B. um einen Aufsatz } \\
\text { oder ein Referat vorzubereiten) }\end{array}$ & $\begin{array}{l}\text { Nie oder fast nie / ein- } \\
\text { oder zweimal im Monat / } \\
\text { ein- oder zweimal in der } \\
\text { Woche / fast jeden Tag / } \\
\text { jeden Tag }\end{array}$ & $-.20(.92)$ & .83 \\
\hline USESCH & $\begin{array}{l}\text { Wie oft benutzt du in der Schule } \\
\text { den Computer für folgende } \\
\text { Aktivitäten? Für schulische } \\
\text { Aufgaben im Internet surfen }\end{array}$ & $\begin{array}{l}\text { Nie oder fast nie / ein- } \\
\text { oder zweimal im Monat / } \\
\text { ein- oder zweimal in der } \\
\text { Woche / fast jeden Tag / } \\
\text { jeden Tag }\end{array}$ & $-.01(.84)$ & .89 \\
\hline
\end{tabular}


Die positive Einstellung der Schülerinnen und Schüler (ICTATTPOS) dient dabei als abhängige Variable, alle anderen aufgelisteten Variablen als Prädiktoren. Dies sind das Geschlecht (ST04Q01), der sozioökonomische Status (ESCS), die Geräteverfügbarkeit zuhause (ICTHOME) und in der Schule (ICTSCH), die Häufigkeit der Nutzung digitaler Medien für Unterhaltungszwecke zuhause (ENTUSE), für schulische Zwecke zuhause (HOMSCH) und für schulische Zwecke in der Schule (USESCH). Die Skalierung dieser Variablen, bei denen es sich abgesehen vom Geschlecht nicht um Einzelitems, sondern um aus mehreren Items zusammengesetzte Index-Werte handelt, erfolgte auf Basis von Partial-Credit Modellen der Item-Response-Theorie durch das PISA Konsortium. Das genaue Vorgehen bei der Bildung dieser Variablen wird im technischen Bericht von PISA 2012 im Detail erläutert und alle Skalenwerte können wie z-standardisierte Werte interpretiert werden (OECD, 2014). Bei den deskriptiven Statistiken (Tabelle 1) fällt auf, dass Schweizer Schülerinnen und Schüler unterdurchschnittliche Einstellungen zum Lernen mit digitalen Medien aufweisen. Dies obwohl die Geräteverfügbarkeit zuhause und in der Schule überdurchschnittlich hoch ausfällt.

\section{Methoden der Hypothesenprüfung}

$\mathrm{Da}$ davon auszugehen ist, dass die Ausprägung dieser Einzelvariablen und ihrer Zusammenhänge nicht nur von einzelnen Schülerinnen und Schülern, sondern auch von der Zugehörigkeit zu einer bestimmten Schule abhängig ist, wird angesichts der geschachtelten Datenstruktur von einer Notwendigkeit von Multilevel-Analysen ausgegangen. Zur Prüfung der einzelnen Prädiktoren in Bezug auf die Einstellung von Schülerinnen und Schülern wurden deshalb einerseits einfache gewichtete Korrelationen und andererseits lineare gewichtete Multilevel-Regressionsmodelle verwendet. Um korrekte Standardfehler (und p-Werte) zu erhalten, wurden die Berechnungen mit Funktionen aus dem R-Package BIFIEsurvey 1.11-0 durchgeführt (BIFIE.data.jack, BIFIE. correl, BIFIE.twolevelreg). Diese Funktionen beziehen die Replicated und Final Weights auf Schülerebene und die Final Weights auf Schulebene mit ein, die aufgrund des komplexen Samplings der vorliegenden PISA-Daten nötig sind. Aufgrund theoretischer Überlegungen wurde dabei ein Random-Intercept-Random-Slope-Modell geprüft. Erstens ist anzunehmen, dass Schülerinnen und Schüler einer Schule sich in ihren Einstellungen bezüglich digitaler Lernmedien ähneln, was zu unterschiedlichen mittleren Grundstimmungen an verschiedenen Schulen führen kann. Dies wird durch den Random Intercept für das Gesamtmodell berücksichtigt. Zweitens ist anzunehmen, dass die Art und Weise der Mediennutzung innerhalb von Schulen ähnlich ist, so dass die gesamtschulische Häufigkeit der Mediennutzung in unterschiedlichen Schulen einen unterschiedlichen Einfluss auf die Einstellungen der Schülerinnen und Schüler haben könnte. Somit wird die computerbezogene positive Einstellung (ICTATTPOS) in folgendem Modell vorhergesagt durch einen bestimmten Ausgangswert 
(Intercept) sowie die Kombination der Variablen Geschlecht (ST04Q01), sozioökonomischer Status (ESCS), Geräteverfügbarkeit zuhause (ICTHOME) und in der Schule (ICTSCH), Entertainment-bezogene Nutzung (ENTUSE), Nutzung digitaler Medien zuhause für die Schule (HOMSCH) sowie schulische Nutzung (USESCH). Dabei können sowohl der Einfluss des Intercept als auch der Einfluss der Variable der schulischen Nutzung (USESCH) je nach Schule variieren. Die USESCH Variable wurde also mit einem zufälligen Intercept und einem zufälligen Slope in das Modell mit einbezogen. Der Grund hierfür ist die Annahme, dass sich hier je nach Art der schulischen Nutzung unterschiedliche statistische Effekte ergeben dürften und dass sich die Nutzung hier zwischen Schulen unterscheidet. Bei der Berechnung dieses Random-Intercept-Random-Slope-Modells wird zudem auf Level 1 für die Schüler die Gewichtungsvariable der Student Replicate Weights und auf Level 2 für Schulen die schulbezogene Gewichtungsvariable (W_FSCHWT) berücksichtigt. Die Variable W_FSCHWT wurde dafür aus dem Schulfragebogen entnommen und mit dem Schülerdatensatz kombiniert. Die Modellspezifikation lautet

ICTATTPOSij $=\mathrm{b} 0 \mathrm{j}+\mathrm{b} 1 \mathrm{j}^{*}$ USESCHij $+\mathrm{b} 2{ }^{*}$ ICTSCHij $+\mathrm{b} 3^{*}$ ENTUSEij + b4*HOMSCHij + b5 ${ }^{*}$ ICTHOMEij + b6*ST04Q01ij + b7*ESCSij + rij

wobei sich Schulen in Bezug auf das Einstellungsniveau der Schülerinnen und Schüler unterscheiden dürften (Random Intercept), d.h.

$\mathrm{b} 0 \mathrm{j}=\mathrm{g} 00+\mathrm{u} 0 \mathrm{j}$

und sich auch der Einfluss der schulischen Mediennutzung nach Schulzugehörigkeit unterscheiden dürfte (Random Slope), d.h.

$\mathrm{b} 1 \mathrm{j}=\mathrm{g} 10+\mathrm{u} 1 \mathrm{j}$.

Für die Analysen wird ein Signifikanzniveau von 5\% zugrunde gelegt.

\section{Ergebnisse}

Vor Prüfung des beschriebenen Modells werden die Zusammenhänge zwischen den einbezogenen Variablen zunächst mithilfe einfacher Korrelationen geprüft (Tabelle 2, nichtsignifikante Zusammenhänge grau markiert). Als Gewichtungsvariable wurde dabei das Final Student Weight (W_FSTUWT) verwendet. Dabei zeigt sich, dass insbesondere die häusliche Computernutzung für schulische Zwecke moderat mit den computerbezogenen Einstellungen korreliert. Immer noch signifikant, jedoch deutlich schwächer fallen die Zusammenhänge zwischen unterrichtlicher Nutzung und freizeitlicher Nutzung auf der einen Seite und Einstellungen auf der anderen Seite aus. Nur geringe direkte Zusammenhänge mit den Einstellungen bestehen bei den Geräteverfügbarkeiten zuhause und in der Schule, noch geringere beim sozioökonomischen Status und 
beim Geschlecht, wo der Zusammenhang gegen Null tendiert. Weitere interessante und positiv moderate Korrelationen existieren zwischen häuslicher Freizeitnutzung und häuslicher Nutzung digitaler Medien für schulische Zwecke, sowie zwischen schulischer Computernutzung und häuslicher Computernutzung für schulische Zwecke. Nur eine äusserst schwache direkte Korrelation ergibt sich dabei jedoch in Bezug auf schulische Computernutzung und häusliche Computernutzung für Freizeitzwecke.

Tabelle 2: Gewichtete bivariate Korrelationen (Pearson's r) zwischen den Variablen des Modells

\begin{tabular}{lrcccccr}
\hline & $\begin{array}{r}\text { ICT } \\
\text { ATTPOS }\end{array}$ & $\begin{array}{r}\text { ICT } \\
\text { HOME }\end{array}$ & $\begin{array}{r}\text { ICT } \\
\text { SCH }\end{array}$ & $\begin{array}{r}\text { ENT } \\
\text { USE }\end{array}$ & $\begin{array}{r}\text { HOM } \\
\text { SCH }\end{array}$ & $\begin{array}{r}\text { USE } \\
\text { SCH }\end{array}$ & 4Q01 \\
\hline ICTHOME & 0.14 & & & & & & \\
ICTSCH & 0.11 & 0.17 & & & & & \\
ENTUSE & 0.22 & 0.33 & 0.06 & & & & \\
HOMSCH & 0.35 & 0.17 & 0.20 & 0.31 & & & \\
USESCH & 0.20 & 0.16 & 0.35 & 0.26 & 0.43 & & \\
ST04Q01 & 0.00 & 0.15 & 0.02 & 0.21 & -0.05 & 0.03 & \\
ESCS & 0.07 & 0.14 & -0.06 & 0.01 & 0.08 & -0.04 & -0.02 \\
\hline
\end{tabular}

Im Mehrebenenmodell zeigt sich, dass alle einbezogenen Variablen (mit Ausnahme des Geschlechts) signifikante fixe Faktoren zur Vorhersage der Einstellungen von Schülerinnen und Schülern zum Lernen mit ICT darstellen (Tabelle 3). Wie in den einfachen Korrelationen wird auch hier deutlich, dass in der Kombination aller Faktoren, insbesondere die häusliche Nutzung digitaler Medien für schulische Zwecke, die grösste statistische Vorhersagekraft in Bezug auf die diesbezüglichen Einstellungen besitzt $(b=.31)$, gefolgt von häuslicher Nutzung für Unterhaltungszwecke $(\mathrm{b}=.13)$. Die schulische Nutzung hat demgegenüber nur noch einen kleinen statistischen Effekt $(b=.05)$. Beachtlich ist auch hier, dass das Geschlecht kein signifikanter Prädiktor für die Einstellungen ist und auch der sozioökonomische Status einen vergleichsweise kleinen Einfluss besitzt. Da das Modell auf standardisierten Variablen beruht, lassen sich die Regressionskoeffizienten näherungsweise als standardisierte Effekte interpretieren. Dass auch kleine Effektstärken noch signifikant sind, ist vor allem auf die sehr grosse Stichprobe zurückzuführen und sollte nicht darüber hinwegtäuschen, dass die Erklärungskraft in Bezug auf die abhängige Variable sehr klein ist. 
Tabelle 3: Feste Effekte des Multilevel-Regressionsmodells auf die abhängige Variable ICTATTPOS

\begin{tabular}{lrrr}
\hline & $\mathrm{b}$ & $\mathrm{SE}$ b & $\mathrm{p}$ \\
\hline Intercept (g00) & -0.26 & 0.04 & $* * *$ \\
ICT-Verfügbarkeit Zuhause (ICTHOME / b5) & 0.05 & 0.02 & $* *$ \\
ICT-Verfügbarkeit in der Schule (ICTSCH / b2) & 0.03 & 0.01 & $* *$ \\
ICT-Nutzung zuhause für Unterhaltungszwecke (ENTUSE / b3) & 0.13 & 0.02 & $* * *$ \\
ICT-Nutzung zuhause für schulische Zwecke (HOMSCH / b4) & 0.31 & 0.02 & $* * *$ \\
ICT-Nutzung in der Schule für schulische Zwecke (USESCH / g10) & 0.05 & 0.02 & $*$ \\
Geschlecht (ST04Q01 / b6) & -0.02 & 0.02 & n.s. \\
Sozioökonomischer Status (ESCS / b7) & 0.06 & 0.01 & $* * *$ \\
\hline
\end{tabular}

${ }^{*} \mathrm{p} \leq .05,{ }^{* *} \mathrm{p} \leq .01,{ }^{* * *} \mathrm{p} \leq .001$

Die Berücksichtigung der Mehrebenenstruktur zeigt zudem keine grossen Effekte für die Schulzugehörigkeit (Tabelle 4). Die Varianz des Intercepts ist zwar signifikant, jedoch im Umfang eher klein. D.h. Schulen unterscheiden sich mit einer durchschnittlichen Standardabweichung von $\mathrm{SD}=.14^{*}$ bezüglich der theoretisch anzunehmenden Ausgangseinstellung der Schülerinnen und Schüler. Die Varianz des variablen Effekts USESCH ist mit einer Standardabweichung von $\mathrm{SD}=.07$ ebenfalls gering und nicht signifikant (Tabelle 4), so dass sich die Schulen statistisch nicht bezüglich der Stärke des Zusammenhangs zwischen USESCH und ICTATTPOS unterscheiden.

Tabelle 4: Variable Effekte des Multilevel-Regressionsmodells

\begin{tabular}{lccc}
\hline & Wert & SE & p \\
\hline Varianz Intercept (var(u0j)) & 0.02 & 0.01 & $*$ \\
Korrelation Intercept, USESCH (cor(u0j, u1j)) & 0.66 & 0.34 & $*$ \\
Varianz USESCH (var (u1j)) & 0.01 & 0.01 & n.s. \\
Residualvarianz (var(rij)) & 0.88 & 0.01 & $* * *$ \\
\hline
\end{tabular}

${ }^{*} \mathrm{p} \leq .05,{ }^{* *} \mathrm{p} \leq .01,{ }^{* * *} \mathrm{p} \leq .001$

Allerdings ist eine deutliche Korrelation zwischen den Varianzen des Intercepts und des Einflusses der schulischen Computernutzung sichtbar (cor(u0j, u1j): r $=.66)$. Bei der Mehrebenenanalyse wird aber nur etwa zwei Prozent der Varianz in der abhängigen Variable durch die Zugehörigkeit zu einer bestimmten Schule erklärt (ICC Conditional $=.02$ ). Das gesamte Modell besitzt ausserdem nur 
eine moderate Varianzaufklärung von $\mathrm{R}^{2}=.23$ auf Schülerebene, von $\mathrm{R}^{2}=.34$ auf Schulebene und eine totale Varianzaufklärung von $\mathrm{R}^{2}=.23$ (Snijders \& Bosker, 2012).

Die Hypothesen $\mathrm{H} 1$ und $\mathrm{H} 2$ können damit als bestätigt gelten. Die dabei angenommene Bedeutung der Kontrollvariablen lässt sich für den sozioökonomischen Status bestätigen, nicht jedoch für das Geschlecht. Bezüglich Hypothese H3 lässt sich feststellen, dass die Zugehörigkeit zu einer bestimmten Schule sich zwar in kleinen Unterschieden in den Einstellungen bemerkbar macht, sich der Einfluss der Unterrichtsnutzung digitaler Medien auf die diesbezüglichen Einstellungen jedoch nicht zwischen Schulen unterscheidet.

\section{Zusammenfassung und Diskussion}

Die Ergebnisse führen zu dem Schluss, dass vor allem die häusliche Nutzung von digitalen Geräten für schulische Zwecke als deutlicher und positiver Prädiktor für die Einstellungen von Schülerinnen und Schülern zum Lernen mit digitalen Medien sind. Die schulische Nutzung im Unterricht zeigt daneben zwar ebenfalls einen positiven, in Kombination jedoch vergleichsweise geringfügigen Effekt, ebenso wie die Geräteverfügbarkeiten für die Schülerinnen und Schüler zuhause und in der Schule. Dass auch die private Nutzung für Unterhaltungszwecke einen positiven Effekt aufweist, kann mit globalen Effekten der Medienpräferenz und der Medienaffinität der Schülerinnen und Schüler zu tun haben. Ob Schülerinnen und Schüler also die Nutzung von ICT für Lernzwecke als sinnvoll einschätzen, lässt sich auf Basis dieser Ergebnisse vor allem auf ihre häuslichen Lernerfahrungen mit digitalen Medien zurückführen und weniger auf die schulischen Erfahrungen. Die Berücksichtigung der Multilevel-Struktur zeigte zudem, dass es hier auch kaum Unterschiede zwischen Schulen gibt. Solche Befunde könnten auch damit zusammenhängen, dass die Schweiz immer noch zu den Ländern mit einer im Schnitt eher seltenen schulischen Computernutzung gehört (OECD, 2015) und manche Zusammenhänge in Ländern mit besonders häufiger schulischer Computernutzung möglicherweise höher ausfallen könnten (OECD, 2016). In künftigen Studien wäre deshalb auch ländervergleichend zu prüfen, ob sich ähnliche Zusammenhänge auch in Ländern mit häufigerer schulischer Computernutzung ergeben oder ob hier der Einfluss der schulischen Nutzung höher ist. Dass der Effekt bei der häuslichen Mediennutzung für schulische Zwecke grösser ist als bei der schulischen Mediennutzung selbst, muss jedoch zu denken geben - einerseits vor dem Hintergrund der hohen Erwartungen, die mit schulischer Mediennutzung verbunden sind und andererseits vor dem Hintergrund der unterschiedlichen häuslichen Lernmilieus, welches Schülerinnen und Schüler vorfinden. Seit den Anfängen der PISA-Studien wurde immer wieder nachgewiesen, dass Schulen häusliche Bildungsvoraussetzungen oft nicht ausgleichen können (Hopfenbeck et al., 2017). Auch wenn es deshalb 
nicht erstaunt, dass dies in Bezug auf die Einstellungen zu digitalen Medien nicht anders ist, wäre es sicherlich wünschenswert, wenn Schulen diesbezüglich stärker ausgleichend wirken könnten. Geschlechtsspezifische Einflüsse zeigen sich in der Schweiz allerdings erstaunlicherweise nicht, und die Effekte des sozioökonomischen Status sind gering, im Gegensatz zu vielen anderen Befunden aus anderen Ländern (Meelissen, 2008). Ein möglicher Grund liegt im Fokus auf die lernbezogenen Computereinstellungen, wo sich die Interessen offenbar nicht so deutlich unterscheiden wie bei den meisten anderen Studien, in denen das allgemeine Computerinteresse im Mittelpunkt steht. Bei alledem muss zudem darauf hingewiesen werden, dass alle Variablen zusammengenommen nur etwa ein Viertel der Varianz in den Einstellungen der Schülerinnen und Schüler erklären. Das heisst, dass künftige Studien noch andere mögliche Effekte einbeziehen müssen, u.a. die beschriebenen weiteren Prädiktoren, die in Technologieakzeptanzmodellen verwendet werden und die vielleicht in künftigen Begleitbefragungen Eingang in den Fragebogen finden könnten, oder auch die allgemeinen, nicht speziell computerbezogenen, Einstellungen zu schulischem Lernen, die sich schon heute in den PISA Begleitbefragungen finden. Die Befunde können nichtsdestotrotz schon jetzt praktische Bedeutsamkeit haben. Wenn es bei der Entwicklung positiver lernbezogener Einstellungen vor allem auch auf häusliche Praktiken ankommt, dann sind damit Kinder und Jugendliche benachteiligt, die zuhause hier nicht genügend Anregungen erhalten. Schulen sollten deshalb versuchen, die häusliche Nutzung digitaler Technologien für schulische Zwecke neben einem häufigeren schulischen Einsatz verstärkt zu fördern.

Bei der Interpretation solcher Befunde müssen allerdings verschiedene Einschränkungen berücksichtigt werden. Die wichtigste Einschränkung der Studie liegt in dem Umstand, dass hier die Einstellungen zur Qualität des Lernens mit digitalen Medien durch die Quantität und nicht nur die Qualität ihrer Nutzung erklärt werden. In den PISA Befragungen fehlen bisher Fragen zur didaktischen, fachdidaktischen und mediendidaktischen Qualität der Computernutzung, und es ist ohnehin schwierig, solche Qualitäten mittels Befragungen abzufragen (Petko, 2012). Eine weitere wichtige Einschränkung liegt in dem Umstand, dass bei PISA lediglich querschnittliche Daten vorliegen. Damit kann die Kausalität der untersuchten Zusammenhänge nicht zweifelsfrei festgestellt werden. Anders gesprochen: $\mathrm{Ob}$ tatsächlich die Nutzungshäufigkeit die Einstellung beeinflusst und nicht auch oder eher umgekehrt, könnte nur in längsschnittlichen oder experimentellen Forschungsdesigns geprüft werden. Weitere Einschränkungen liegen im Umstand, dass neben Geschlecht und sozioökonomischen Faktoren nur medienbezogene Prädiktorvariablen in das Modell einbezogen wurden. So ist es durchaus denkbar, dass auch andere Variablen einen wesentlichen Einfluss haben könnten. Es könnte etwa die allgemeine Schul- und Lernmotivation auch einen Einfluss darauf haben, ob digitale Medien ein gutes Lernwerkzeug darstellen. Trotz dieser Einschränkung hat die vorliegende Studie den Versuch gemacht, Einstellungen zum Lernen mit 
digitalen Medien als Resultat schulischer und ausserschulischer Medienerfahrungen zu verstehen. Es kann davon ausgegangen werden, dass solche Einstellungen die Grundlage dafür bieten, ob und wie Schülerinnen und Schüler solche Technologien als sinnvolles Instrument des lebenslangen Lernens einsetzen. Die Förderung positiver Einstellungen zum Lernen mit digitalen Technologien ist vor diesem Hintergrund ein wichtiges Bildungsziel.

\section{Literatur}

Bohner, G. \& Dickel, N. (2011). Attitudes and attitude change. Annual review of psychology, $62,391-417$.

Cheung, A. C. K. \& Slavin, R. E. (2012). How features of educational technology applications affect student reading outcomes: A meta-analysis. Educational Research Review, 7, 198-215. doi:10.1016/j.edurev.2012.05.002

Cheung, A. C. K. \& Slavin, R. E. (2013). The effectiveness of educational technology applications for enhancing mathematics achievement in K-12 classrooms: A meta-analysis. Educational Research Review, 9, 88-113. doi:10.1016/j.edurev.2013.01.001

Davis, F. D. (1989). Perceived usefulness, perceived ease of use, and user acceptance of information technology. MIS Quarterly, 19, (2), 319-340. doi:10.2307/249008

Delors, J. (1998). Education for the twenty-first century: issues and prospects. Paris, France: UNESCO.

Fishbein, M. \& Ajzen, I. (1977). Belief, attitude, intention, and behavior: An introduction to theory and research. Reading, MA: Addison-Wesley.

Fishbein, M. \& Ajzen, I. (2010). Predicting and changing behavior: The reasoned action approach. New York, NY: Taylor \& Francis.

Gerick, J. \& Eickelmann, B. (2015). Einsatz digitaler Medien im Mathematikunterricht und Schülerleistungen. Ein internationaler Vergleich von Bedingungsfaktoren auf Schulebene auf der Grundlage von PISA 2012. Tertium Comparationis. Journal für International und Interkulturell Vergleichende Erziehungswissenschaft, 20, (2), 152-181.

Hatzigianni, M., Gregoriadis, A. \& Fleer, M. (2016). Computer use at schools and associations with social-emotional outcomes - A holistic approach. Findings from the longitudinal study of Australian Children. Computers \& Education, 95, 134-150. doi:10.1016/j. compedu.2016.01.003

Hopfenbeck, T. N., Lenkeit, J., Masri, Y. E., Cantrell, K., Ryan, J. \& Baird, J.-A. (2017). Lessons Learned from PISA: A Systematic Review of Peer-Reviewed Articles on the Programme for International Student Assessment. Scandinavian Journal of Educational Research, 1-21. doi:10.1080/00313831.2016.1258726

House, J. (2007). Relationships between computer use and reading achievement of elementary-school students: Results from the PIRLS 2001 assessment. International Journal of Instructional Media, 34, (4), 449-456.

Howard, S. K., Ma, J. \& Yang, J. (2016). Student rules: Exploring patterns of students' computer-efficacy and engagement with digital technologies in learning. Computers \& Education, 101, 29-42. doi:10.1016/j.compedu.2016.05.008

Huber, M. \& Ramseier, E. (2002). Vertrautheit im Umgang mit Computern. In BFS \& EDK (Hrsg.), Für das Leben gerüstet? Die Grundkompetenzen der Jugendlichen - Kurzfassung des nationalen Berichts PISA 2000 (S. 53-63). Neuchâtel, Schweiz: Bundesamt für Statistik.

Jones, C., Ramanau, R., Cross, S. \& Healing, G. (2010). Net generation or Digital Natives: Is there a distinct new generation entering university? Computers \& Education, 54, (3), 722-732. doi:10.1016/j.compedu.2009.09.022

Lee, Y.-H. \& Wu, J.-Y. (2012). The effect of individual differences in the inner and outer states of ICT on engagement in online reading activities and PISA 2009 reading literacy: 
exploring the relationship between the old and new reading literacy. Learning and Individual Differences, 22, (3), 336-342. doi:10.1016/j.lindif.2012.01.007

Levin, T. \& Wadmany, R. (2005). Changes in educational beliefs and classroom practices of teachers and students in rich technology-based classrooms [1]. Technology, Pedagogy and Education, 14, (3), 281-307. doi:10.1080/14759390500200208

Li, Q. (2007). Student and teacher views about technology: A tale of two cities? Journal of research on Technology in Education, 39, (4), 377-397. doi:10.1080/15391523.2007.107 82488

Lorenz, R. \& Gerick, J. (2014). NeueTechnologien und die Leseleistung von Grundschulkindern. Zur Bedeutung der schulischen und außerschulischen Nutzung digitaler Medien. In B. Eickelmann, R. Lorenz, M. Vennemann, J. Gerick, \& W. Bos (Hrsg.), Grundschule in der digitalen Gesellschaft. Befunde aus den Schulleistungsstudien IGLU und TIMSS 2011 (S. 59-71). Münster, Deutschland: Waxmann.

Meelissen, M. (2008). Computer attitudes and competencies among primary and secondary school students. In J. Voogt \& G. Knezek (Hrsg.), International handbook of information technology in primary and secondary education (S. 381-395). Berlin, Deutschland: Springer. doi:10.1007/978-0-387-73315-9_68

Naumann, J. \& Sälzer, C. (2017). Digital reading proficiency in German 15-year olds: Evidence from PISA 2012. Zeitschrift für Erziehungswissenschaft, First Online, 1-19. doi:10.1007/s11618-017-0758-y

Oblinger, D. G. \& Oblinger, J. L. (2005). Is it age oder IT: First steps toward understanding the net generation. In D. G. Oblinger \& J. L. Oblinger (Ed.), Educating the Net Generation (pp. 2.1-2.20). EDUCAUSE. Retrieved from http://net.educause.edu/ir/ library/pdf/pub7101.pdf

OECD. (2014). PISA 2012 Technical Report. Paris, France: OECD Publishing. Retrieved from http://www.oecd.org/pisa/pisaproducts/PISA-2012-technical-report-final.pdf

OECD. (2015). Students, Computers and Learning: Making the Connection. Paris, France: PISA OECD Publishing. doi:10.1787/9789264239555-en

OECD. (2016). PISA 2015 Results (Volume I): Excellence and Equity in Education? Paris, France: PISA, OECD Publishing. doi:10.1787/9789264266490-en

OECD. (2017). PISA 2015 Technical Report. Paris, France: OECD Publishing. Retrieved from https://www.oecd.org/pisa/sitedocument/PISA-2015-technical-report-final.pdf

Papanastasiou, E. \& Paparistodemou, E. (2007). Examining educational technology and achievement through latent variable modeling. In T. Loveless (Ed.), Lessons learned: What international assessments tell us about Math achievement (pp. 205-225). Washington, D.C.: Brookings Institution Press.

Petko, D. (2012). Hemmende und förderliche Faktoren des Einsatzes digitaler Medien im Unterricht: Empirische Befunde und forschungsmethodische Probleme. In R. SchulzZander, B. Eickelmann, H. Moser, H. Niesyto, \& P. Grell (Hrsg.), Jahrbuch Medienpädagogik 9 (S. 29-50). Wiesbaden: Springer. doi:10.1007/978-3-531-94219-3_3

Petko, D., Cantieni, A. \& Prasse, D. (2016). Perceived Quality of Educational Technology Matters A Secondary Analysis of Students' ICT Use, ICT-Related Attitudes, and PISA 2012 Test Scores. Journal of Educational Computing Research, 50, (8),1070-1091. doi:10.1177/0735633116649373

Prasse, D. \& Egger, N. (2016, Juni). Einstellungen von Primarschüler/innen zum Lernen mit digitalen Medien in Klassen mit und ohne Tablets. Zur Rolle von Lernaktivitäten, Lehrpersonen, Eltern und Peers. Vortrag auf dem Kongress der Schweizerischen Gesellschaft für Bildungsforschung (SGBF), Universität Lausanne.

Prasse, D., Egger, N. \& Döbeli Honegger, B. (2017). Mobiles Lernen. Auch zu Hause? In J. Bastian \& S. Aufenanger (Hrsg.), Tablets in Schule und Unterricht (S. 209-239). Wiesbaden, Deutschland: Springer Fachmedien. 
Ramseier, E. \& Holzer, T. (2005). Vertrautheit mit Informations- und Kommunikationstechnologien (IKT). In C. Zahner Rossier (Hrsg.), PISA 2003: Kompetenzen für die Zukunft. Zweiter nationaler Bericht (S. 119-130). Neuchâtel, Schweiz: Bundesamt für Statistik.

Reimann, P. \& Aditomo, A. (2013). Technology-supported learning and academic achievement. In J. Hattie \& E. M. Anderman (Ed.), International guide to student achievement (pp. 399-401). New York, NY: Routledge.

Rychen, D. S. \& Salganik, L. H. (2003). Key competencies for a successful life and a well-functioning society. Cambridge, MA: Hogrefe \& Huber.

Salvisberg, S. M. \& Zampieri. (2014). Vertrautheit mit Informations- und Kommunikationstechnologien (ICT). In Konsortium PISA.ch (Hrsg.), PISA 2012. Vertiefende Analysen (S. 49-58). Neuchâtel, Schweiz: SBFI/EDK.

Schulmeister, R. (2009). Gibt es eine "Net Generation"? Erweiterte Version 3.0. Universität Hamburg. Retrieved from http://epub.sub.uni-hamburg.de/epub/volltexte/2013/19651/

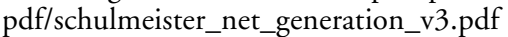

Shewbridge, C., Ikeda, M. \& Schleicher, A. (2005). Are students ready for a technology-rich world? What PISA studies tell us. Paris, France: OECD.

Skryabin, M., Zhang, J., Liu, L. \& Zhang, D. (2015). How the ICT development level and usage influence student achievement in reading, mathematics, and science. Computers and Education, 85, 49-58. doi:10.1016/j.compedu.2015.02.004

Snijders, T. \& Bosker, R. (2012). Multilevel Analysis: An Introduction to Basic and Advanced Multilevel Modeling (2th ed.). London, England: Sage.

Tamim, R. M., Bernard, R. M., Borokhovski, E., Abrami, P. C. \& Schmid, R. F. (2011). What forty years of research says about the impact of technology on learning : A secondorder meta-analysis and validation study. Review of Educational Research, 81, (1), 4-28. doi: $10.3102 / 0034654310393361$

Tapscott, D. (1998). Growing up digital: The rise of the Net Generation. New York, NY: McGraw-Hill.

Venkatesh, V., Thong, J. Y. \& Xu, X. (2012). Consumer acceptance and use of information technology: extending the unified theory of acceptance and use of technology. MIS quarterly, 36, (1), 157-178.

Woessmann, L. \& Fuchs, T. (2007). Computers and student learning: Bivariate and multivariate evidence on the availability and use of computers at home and at school. CESifo Working Paper Series, 1321, 1-34. Retrieved from http://papers.ssrn.com/sol3/papers. cfm?abstract_id=619101

Young, B. J. (2000). Gender differences in student attitudes toward computers. Journal of Research on Computing in Education, 33, (2), 204-216. doi:10.1080/08886504.2000.1 0782310

Schlagworte: PISA, Large Scale Assessment, Computernutzung, Medien, Einstellungen, Überzeugungen 


\title{
Qu'est-ce qui influence les attitudes des élèves à l'égard de l'apprentissage par les technologies numériques? Analyse de I'enquête PISA 2012 auprès des étudiants en Suisse
}

\begin{abstract}
Résumé
Les élèves devraient non seulement apprendre à connaître les technologies numériques à l'école en tant qu'outil d'apprentissage, mais aussi, idéalement, développer des attitudes positives envers l'apprentissage par ces technologies. La mesure dans laquelle les écoles contribuent effectivement à cet effort est vérifiée sur la base de l'échantillon suisse de l'enquête PISA 2012 auprès des étudiants. Les analyses à plusieurs niveaux montrent que l'utilisation des technologies numériques à la maison pour les besoins de l'école a un rapport substantiel avec les attitudes des élèves, tandis que l'utilisation des technologies numériques à l'école n'a qu'un effet minime. Cet effet peut s'expliquer par l'utilisation relativement rare des technologies numériques dans les écoles suisses. Compte tenu de l'égalité des chances, il est d'une grande importance que les écoles deviennent plus actives à cet égard.
\end{abstract}

Mots-clés: PISA, évaluation à grande échelle, utilisation de l'ordinateur, technologie éducative, attitudes, croyances, PISA

\section{Cosa influenza gli atteggiamenti degli studenti e delle studentesse nei confronti dell'apprendimento con le tecnologie digitali? Un'analisi del sondaggio PISA 2012 in Svizzera}

\section{Riassunto}

Gli studenti e le studentesse non dovrebbero soltanto conoscere le tecnologie digitali a scuola come strumenti di apprendimento, ma idealmente dovrebbero anche sviluppare atteggiamenti positivi nei confronti dell'apprendimento con queste tecnologie. Quanto le scuole contribuiscano effettivamente a questo sforzo è oggetto di analisi sulla base del campione svizzero del sondaggio PISA 2012 rivolto agli studenti. Le analisi multilivello mostrano che l'uso delle tecnologie digitali a casa per scopi scolastici ha un legame sostanziale con gli atteggiamenti degli alunni, mentre l' uso delle tecnologie digitali a scuola ha solo un effetto limitato. Questo effetto potrebbe essere spiegato dall'uso relativamente raro delle tecnologie digitali nelle scuole svizzere. Tenendo in considerazione le pari opportunità, si sostiene che le scuole debbano diventare più attive a questo proposito.

Parole chaive: PISA, valutazione su larga scala, uso del computer, tecnologia educativa, attitudini, credenze 


\section{What Influences Students' Attitudes Towards Learning with Digital Technologies? An Analysis of the PISA 2012 Student Survey in Switzerland}

\section{Summary}

Students should not only get to know digital technologies in school as a learning tool, but should ideally also develop positive attitudes towards learning with these technologies. The extent to which schools actually make a contribution to this endeavor is examined on the basis of the Swiss sample of the PISA 2012 student survey. Multi-level analyses show that the use of digital technologies at home for school purposes has a substantial connection with the attitudes of pupils, while the use of digital technologies at school has only a small effect. This effect can possibly be explained by the comparatively rare use of digital technologies in Swiss schools. Considering equal opportunities, it is argued that schools need to become more active in this regard.

Keywords: PISA, Large Scale Assessment, Computer Use, Educational Technology, Attitudes, Beliefs 